\title{
Influence of the Content and Nature of the Plasticizer on the Properties of Butadiene-Nitrile Rubber
}

\author{
Valeriya V. Pavlova, \\ Marina D. Sokolova and Aytalina F. Fedorova \\ Institute of Oil and Gas Problems SB RAS \\ FRC" Yakut Science Center" \\ Yakutsk, Russian Federation
}

Received 22.10.2020, received in revised form 16.01.2021, accepted 21.03.2021

\begin{abstract}
In this article investigates the effect of the content of plasticizers on the properties of rubbers based on rubber BNKS-18 AMN. The traditional, widely used dioctyl phthalate (DOP), as well as the more modern, environmentally friendly dioctyl terephthalate (DOTP), were used as plasticizers. It has been established that the introduction of plasticizers contributes to the production of rubbers with an improved complex of operational properties, such as elasticity, frost resistance, resistance to the corrosive media SZhR-3. At the same time, according to the research results, it was revealed that the introduction of plasticizers in an amount of $20 \mathrm{phr}$ leads to the most noticeable improvement in these properties. In terms of physicomechanical and low temperature properties, the samples containing DOTP significantly exceed those of the samples containing DOP. Thus, our data showed that the use of DOTP is promising as an alternative to the widely used DOP.
\end{abstract}

Keywords: elastomers, frost resistance, nitrile butadiene rubber, plasticizer, physicomechanical properties. 


\title{
Влияние содержания и природы пластификатора
}

на свойства бутадиен-нитрильной резины

\author{
В. В. Павлова, М.Д. Соколова, А.Ф. Федорова \\ Институт проблем нефти и газа СО РАН \\ ФИЦ «Якутский научный иентр СО РАН» \\ Российская Федерация, Якутск
}

\begin{abstract}
Аннотация. В данной статье исследовано влияние содержания пластификаторов на свойства резин на основе каучука БНКС-18 АМН. В качестве пластификаторов использованы традиционный, широко применяемый диоктилфталат (ДОФ), а также более современный, экологичный диоктилтерефталат (ДОТФ). Установлено, что введение пластификаторов способствует получению резин с улучшенным комплексом эксплуатационных свойств, таких как эластичность, морозостойкость, стойкость к среде СЖР-3. При этом по результатам исследований выявлено, что введение пластификаторов в количестве 20 масс.ч. приводит к наиболее заметному улучшению этих свойств. По физико-механическим и низкотемпературным свойствам образцы, содержащие ДОТФ, заметно превосходят показатели образцов, содержащих ДОФ. Таким образом, полученные нами данные показали перспективность применения ДОТФ в качестве альтернативы широко применяемому ДОФ.
\end{abstract}

Ключевые слова: эластомеры, морозостойкость, бутадиен-нитрильный каучук, пластификатор, физико-механические свойства.

Цитирование: Павлова, В.В. Влияние содержания и природы пластификатора на свойства бутадиен-нитрильной резины / В. В. Павлова, М.Д. Соколова, А. Ф. Федорова // Журн. Сиб. федер. ун-та. Техника и технологии, 2021, 14(2). С. 222-232. DOI: 10.17516/1999-494X-0303

\section{Введение}

Пластификация является одним из важнейших методов модификации свойств полимерных композиций. С развитием производства эластомерных материалов роль пластификаторов, применяемых при их переработке, значительно возросла. Введение пластификатора улучшает технологические свойства, способствует распределению сыпучих ингредиентов в резиновых смесях. Правильный выбор типа и количества пластификатора позволяет существенно повысить эластичность, морозостойкость, негорючесть, снизить теплообразование при многократных деформациях, сохранив при этом высокий уровень прочностных свойств. На выбор пластификаторов влияет ряд нескольких требований: они должны совмещаться с каучуком, обладать высокой химической стойкостью и высокой эффективностью пластифицирующего действия. Кроме того, как химические вещества, с которыми работает человек, они должны быть безопасными: нетоксичными, лишенными запаха и цвета и, наконец, пластификаторы должны иметь низкую стоимость.

Анализ литературных данных показал, что в отечественной резиновой промышленности вплоть до 1970 г. ассортимент пластификаторов и мягчителей был крайне ограниченным, но в последние годы начали внедряться новые современные пластификаторы. Доминирующую часть в производстве пластификаторов составляют эфиры о-фталевой кислоты (фталаты), они занимают более 80 \% рынка российской промышленности. Первое место по выпуску и потреблению занимает диоктилфталат (ДОФ). Он хорошо совмещается с каучуками и обеспечивает

$$
-223-
$$


необходимые физико-механические и диэлектрические свойства резинам. Однако имеет повышенную летучесть и ухудшает огнестойкость резин. Морозостойкость резин обеспечивается лишь до минус $40{ }^{\circ} \mathrm{C}[1]$.

В настоящее время рынок пластификаторов и области их применения находятся под влиянием строгих директив по охране окружающей среды и здоровья человека. Регулирование осуществляется Европейским Советом по пластификаторам и интермедиатам (ECPI - European Councilfor Plasticizers and Intermediates). В результате проведенных испытаний применение ДОФ в Европе снижено в связи с негативным влиянием на организм человека (класс опасности -2) [2]. Поэтому одной из актуальных задач является поиск альтернативы данному пластификатору. В последнее время уделяется особое внимание диоктилтерефталату (ДОТФ) как наиболее перспективному заменителю ДОФ благодаря аналогичным пластифицирующим свойствам при сравнимой стоимости. Также ДОТФ считается экологически безопасным (класс опасности - 3) [3].

В связи с этим представляло интерес рассмотреть возможность замены ДОФ в рецептуре стандартной резиновой смеси на ДОТФ. Цель данной статьи - подбор безопасного и перспективного пластификатора взамен широко применяемому, серийному пластификатору ДОФ, а также проведение сравнительных исследований влияния содержания и природы пластификаторов на эксплуатационные свойства резин на основе бутадиен-нитрильного каучука.

\section{Материалы и методы исследования}

В качестве объекта исследования выбран бутадиен-нитрильный каучук марки БНКС-18 АМН. Изделия на основе данного каучука сочетают высокую маслобензостойкость и удовлетворительную морозостойкость $[4,5]$.

В таблице 1 приведены важнейшие показатели исследуемых пластификаторов ДОФ (Китай) и ДОТФ (Россия, ООО «СИБУР»). Данные приведены в соответствии с паспортами качества исследуемых пластификаторов.

Как известно [6], пластифицирующее действие пластификаторов зависит от химического строения, способности молекул к конформационным изменениям и термодинамического сродства пластификаторов к полимеру. Играет роль также природа атомов, входящих в молекулу, полярность связей, размер и форма молекул пластификатора. Если сравнить химическое стро-

Таблица 1. Сравнительная таблица показателей пластификаторов ДОФ и ДОТФ

Table 1. Comparative table of indicators of plasticizers DOP and DOTP

\begin{tabular}{|c|c|c|c|}
\hline № & Показатель & ДОФ & ДОТФ \\
\hline 1 & Молекулярная формула & $\mathrm{C}_{24} \mathrm{H}_{38} \mathrm{O}_{4}$ & $\mathrm{C}_{24} \mathrm{H}_{38} \mathrm{O}_{4}$ \\
\hline 2 & Молекулярная масса, г/моль & 390,56 & 390,56 \\
\hline 3 & 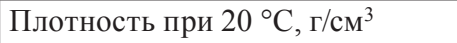 & 0,9852 & 0,9813 \\
\hline 4 & Вязкость при $25^{\circ} \mathrm{C}$, МПа с & 54 & 63 \\
\hline 5 & $\mathrm{~T}_{\mathrm{всп}},{ }^{\circ} \mathrm{C}$ & 205 & 228 \\
\hline 6 & $\mathrm{~T}_{\text {заст }},{ }^{\circ} \mathrm{C}$ & -50 & -48 \\
\hline 7 & Класс опасности & 2 & 3 \\
\hline
\end{tabular}


ение ДОФ и ДОТФ, то они отличаются лишь положением радикала в бензольном кольце, т.е являются структурными изомерами (орто- и пара-изомеры) (рис. 1).

Для оценки эффективности исследуемых пластификаторов разработаны рецептуры резиновых смесей с учетом рекомендованной стандартной смеси [7]. Содержание пластификаторов варьировалось от 0 до 20 масс.ч. на 100 масс.ч. каучука (табл. 2).

Смешивали компоненты на лабораторных вальцах См 350 150/150 (КНР) в течение 40 мин. Вулканизацию образцов проводили при температуре $155^{\circ} \mathrm{C}$ в течение 20 мин на вулканизационном прессе 100-400 2 Э (Россия).

Комплекс исследований пластифицированных резин включал следующие испытания: определение физико-механических свойств (ISO 37-2013), определение коэффициента морозостойкости при растяжении (К $\mathrm{K}_{\mathrm{M}}$ (GOST 408-78), определение твердости по А. Шору (ISO 48 4:2018), определение степени набухания в среде стандартной жидкости СЖР-3 (ISO 1817:2016).

Структурные исследования проведены с помощью растрового электронного микроскопа JEOL JSM 7800F (Япония) с ускоряющим напряжением в 2кВ. Исследование методом растровой электронной микроскопии (РЭМ) осуществлено на низкотемпературных сколах образцов резин при помощи вторичных электронов.

Динамические испытания резин проведены на приборе RPA 2000 фирмы «AlphaTechnologies». Динамическое нагружение вулканизатов при разных частотах (от 0,1 до 20 Гц) проведено при температуре $80{ }^{\circ} \mathrm{C}$ и амплитуде деформации $10 \%$.

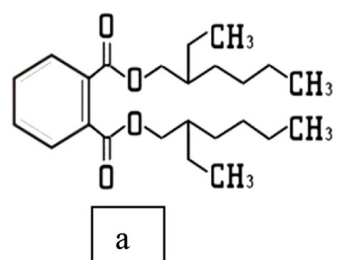<smiles>CCCCC(CC)COC(=O)C1CCC2CCCC2(C(=O)OCC(CC)CCCC)CC1</smiles>

Рис. 1. Химическое строение пластификаторов: а - ДОФ; б - ДОТФ

Fig. 1. Chemical structure of plasticizers: a-DOP; б-DOTP

Таблица 2. Рецептуры резиновых смесей

Table 2. Rubber compounding formulations

\begin{tabular}{|c|l|c|c|c|c|c|c|c|}
\hline \multirow{2}{*}{ № } & \multirow{2}{*}{ Ингредиенты } & \multicolumn{7}{|c|}{ Массовые части на 100 масс. ч. каучука } \\
\cline { 3 - 9 } & & 1 & 2 & 3 & 4 & 5 & 6 & 7 \\
\hline 1 & БНКС-18 АМН & 100 & 100 & 100 & 100 & 100 & 100 & 100 \\
\hline 2 & ТУ 550 & 50 & 50 & 50 & 50 & 50 & 50 & 50 \\
\hline 3 & Пластификатор ДОФ & - & 10 & 15 & 20 & - & - & - \\
\hline 4 & Пластификатор ДОТФ & - & - & - & - & 10 & 15 & 20 \\
\hline 5 & Оксид цинка & 1,5 & 1,5 & 1,5 & 1,5 & 1,5 & 1,5 & 1,5 \\
\hline 6 & Стеариновая кислота & 1,5 & 1,5 & 1,5 & 1,5 & 1,5 & 1,5 & 1,5 \\
\hline 7 & Сера & 2,0 & 2,0 & 2,0 & 2,0 & 2,0 & 2,0 & 2,0 \\
\hline 8 & Каптакс & 5,0 & 5,0 & 5,0 & 5,0 & 5,0 & 5,0 & 5,0 \\
\hline
\end{tabular}


Дифференциальный сканирующий калориметр DSC204 HP/1/G Phoenix фирмы «NETZSCH» использовали для определения температуры стеклования образцов.

\section{Результаты и обсуждение}

Результаты исследований физико-механических свойств резин на основе БНКС-18 АМН в зависимости от различного содержания пластификаторов ДОФ и ДОТФ представлены в табл. 3.

Данные табл. 3 показывают, что введение пластификаторов ДОФ и ДОТФ обеспечивает уровень допустимых значений эксплуатационных свойств для уплотнительных резин. Как видим, при введении пластификаторов в количестве 10 масс.ч. показатели условной прочности сопоставимы с показателями образца, в который не был введен пластификатор. С увеличением содержания пластификаторов в резинах наблюдается незначительное снижение прочностных показателей, а также снижение показателей напряжения при $100 \%$-ном удлинении более чем в 2 раза. Предполагается [8], что существенное влияние на механические свойства пластифицированных полимеров оказывает механизм пластификации. Так, введение пластификатора, молекулярно распределенного между цепями полимера, приводит к увеличению свободного объема, пропорционального количеству пластификатора. Таким образом, снижение значений условной прочности опытных образцов резин с увеличением содержания пластификатора является закономерным эффектом. Показано, что пластифицированные образцы имеют существенно более высокие показатели относительного удлинения в отличие от непластифицированного. Известно [9], что введение пластификатора в резиновые смеси в большинстве случаев повышает данный показатель, так как происходит взаимодействие между полярными груп-

Таблица 3. Физико-механические свойства резин на основе БНКС-18 АМН с различным содержанием пластификаторов ДОФ и ДОТФ

Table 3. Physico-mechanical properties of rubbers based on BNKS-18 AMN with different content of DOP and DOTP

\begin{tabular}{|c|c|c|c|c|c|c|c|}
\hline \multirow{2}{*}{$\begin{array}{c}\text { Содержание пластификаторов, } \\
\text { масс.ч. }\end{array}$} & \multicolumn{7}{|c|}{ Обозначение смесей } \\
\hline & 1 & 2 & 3 & 4 & 5 & 6 & 7 \\
\hline ДОФ & \multirow{2}{*}{0} & 10 & 15 & 20 & - & - & - \\
\hline ДОТФ & & - & - & - & 10 & 15 & 20 \\
\hline \multicolumn{8}{|c|}{ Свойства вулканизатов $\left(155^{\circ} \mathrm{C} × 20\right.$ мин $)$} \\
\hline $\mathrm{TS}_{\mathrm{b}}, \mathrm{M \Pi \textrm {a }}$ & 20,0 & 19,4 & 17,1 & 16,9 & 19,8 & 17,6 & 15,7 \\
\hline $\mathrm{S}_{100}$, МПа & 4,5 & 1,9 & 1,7 & 1,7 & 2,2 & 2,1 & 1,5 \\
\hline $\mathrm{E}_{\mathrm{b}}, \%$ & 402 & 516 & 609 & 690 & 633 & 652 & 703 \\
\hline H, Шор A & 69 & 59 & 58 & 55 & 62 & 61 & 56 \\
\hline $\mathrm{K}_{\mathrm{M}}$ при $-30{ }^{\circ} \mathrm{C}$ & 0,22 & 0,37 & 0,42 & 0,44 & 0,42 & 0,44 & 0,51 \\
\hline $\mathrm{K}_{\mathrm{M}}$ при $-40{ }^{\circ} \mathrm{C}$ & 0,06 & 0,21 & 0,23 & 0,26 & 0,22 & 0,23 & 0,27 \\
\hline $\mathrm{Q}, \%\left(70^{\circ} \mathrm{C} \times 72\right.$ ч) & 12,57 & 6,69 & 6,24 & 4,77 & 8,73 & 8,37 & 4,53 \\
\hline
\end{tabular}

Примечание: $\mathrm{S}_{100}$, МПа - условное напряжение при 100\%-ном удлинении; $\mathrm{TS}_{\mathrm{b}}$, МПа - условная прочность; $\mathrm{E}_{\mathrm{b}}, \%$ относительное удлинение; $\mathrm{K}_{\mathrm{M}}$ - коэффициент морозостойкости при растяжении; $\mathrm{Q}, \%$ - степень набухания в среде СЖР-3 при $70{ }^{\circ} \mathrm{C}$ в течении 72 часов; Н, Шор А - твердость по А. Шору. 
пами как самого бутадиен-нитрильного каучука БНКС-18, так и полярных групп пластификаторов ДОФ и ДОТФ, что влияет в целом на эластичность резин. В таких случаях с увеличением содержания пластификатора будут повышаться показатели относительного удлинения. В данной работе наибольшие показатели относительного удлинения наблюдаются у образцов, содержащих 20 масс.ч. пластификатора и ДОФ, и ДОТФ, что в 1,7 раз больше, чем у образца без пластификатора. Твердость образцов по А. Шору при введении пластификаторов с увеличением содержания пластификаторов снижается. Необходимо отметить, что существенного отличия для большинства исследованных физико-механических показателей резин, пластифицированных ДОФ и ДОТФ, при одних и тех же содержаниях введенного пластификатора не наблюдается. По-видимому, это объясняется тем, что они являются структурными изомерами (М=390,6 г/моль). Однако заметное превосходство показателей относительного удлинения образцов, содержащих ДОТФ, над показателями образцов ДОФ возможно объяснить с точки зрения химического строения ДОТФ (рис. 1).

Исследование стойкости резин в среде СЖР-3 показало, что при введении пластификаторов с увеличением содержания пластификатора в образцах снижается степень набухания. Вероятно, это связано с тем, что наличие в составе образцов пластификаторов с более высокой молекулярной массой (M=390 г/моль), чем молекулярная масса рабочей жидкости СЖР (M $\approx 266$ г/моль), препятствует набуханию резин. Так, при введении 10 и 15 масс.ч. пластификатора ДОФ в образцы степень набухания снижается в 1,8 раза, при введении соответствующего количества пластификатора ДОТФ степень набухания - в 1,5 раза, а при введении 20 масс.ч. пластификаторов ДОФ и ДОТФ почти одинаково - в 2,7 раза.

Одним из основных назначений пластификаторов является улучшение низкотемпературных свойств, которые можно оценить с помощью коэффициента морозостойкости. По данным табл. 3 видно, что введение пластификаторов повышает морозостойкость исследуемых образцов резин. Так, коэффициент морозостойкости при минус $30^{\circ} \mathrm{C}$ и при минус $40{ }^{\circ} \mathrm{C}$ у образцов, содержащих пластификаторы ДОФ и ДОТФ, значительно выше по сравнению с образцом без содержания пластификатора. Образцы, пластифицированные ДОТФ, имеют более высокие значения коэффициента морозостойкости, чем образцы, пластифицированные ДОФ. С увеличением содержания пластификаторов в образцах коэффициент морозостойкости увеличивается. У образцов, содержащих 20 масс.ч. пластификаторов ДОФ и ДОТФ, наблюдаются наибольшие показатели коэффициента морозостойкости. Как было отмечено ранее, морозостойкость резин, пластифицированных ДОФ (соответственно и ДОТФ), обеспечивается лишь до минус $40{ }^{\circ} \mathrm{C}$, о чем свидетельствуют полученные данные. При минус $40{ }^{\circ} \mathrm{C}$ коэффициент морозостойкости у всех пластифицированных образцов сохраняется на уровне нормативного значения $\left(K_{\mathrm{M}} \geq 0,2\right)$. Однако анализ термограммы, полученной методом ДСК (рис. 2), показал, что процесс стеклования образцов, содержащих 20 масс.ч. пластификаторов, начинается при минус $39,7^{\circ} \mathrm{C}(Д О Ф)$ и при минус $40,8^{\circ} \mathrm{C}$ (ДОТФ).

Таким образом, выявлено, что введение пластификаторов и ДОФ, и ДОТФ в количестве 20 масс.ч. оптимально для получения резин с улучшенным комплексом эксплуатационных свойств, таких как эластичность, морозостойкость, стойкость к среде СЖР-3.

Введение пластификаторов в резиновые смеси не только улучшает эластические и низкотемпературные свойства резин, но и облегчает их переработку, повышает пластичность, спо-

$$
-227-
$$


собствует равномерному распределению дисперсных ингредиентов, уменьшению разогрева при смешении и снижает опасность подвулканизации. Для подтверждения данной гипотезы проведены исследования микроструктуры с помощью метода РЭМ, а также динамические испытания образцов резин на приборе RPA 2000. На основании вышеполученных результатов были отобраны следующие образцы: образец № 1 (табл. 2) - без содержания пластификатора (исходный образец), образец № 4 - содержащий 20 масс.ч. пластификатора ДОФ, и образец № 7 - содержащий 20 масс.ч. пластификатора ДОТФ. На рис. 3 представлены микрофотографии исследуемых образцов. Сравнительный анализ полученных изображений низкотемпературного скола образцов позволяет сделать следующие выводы. Видно, что поверхность (на сре-

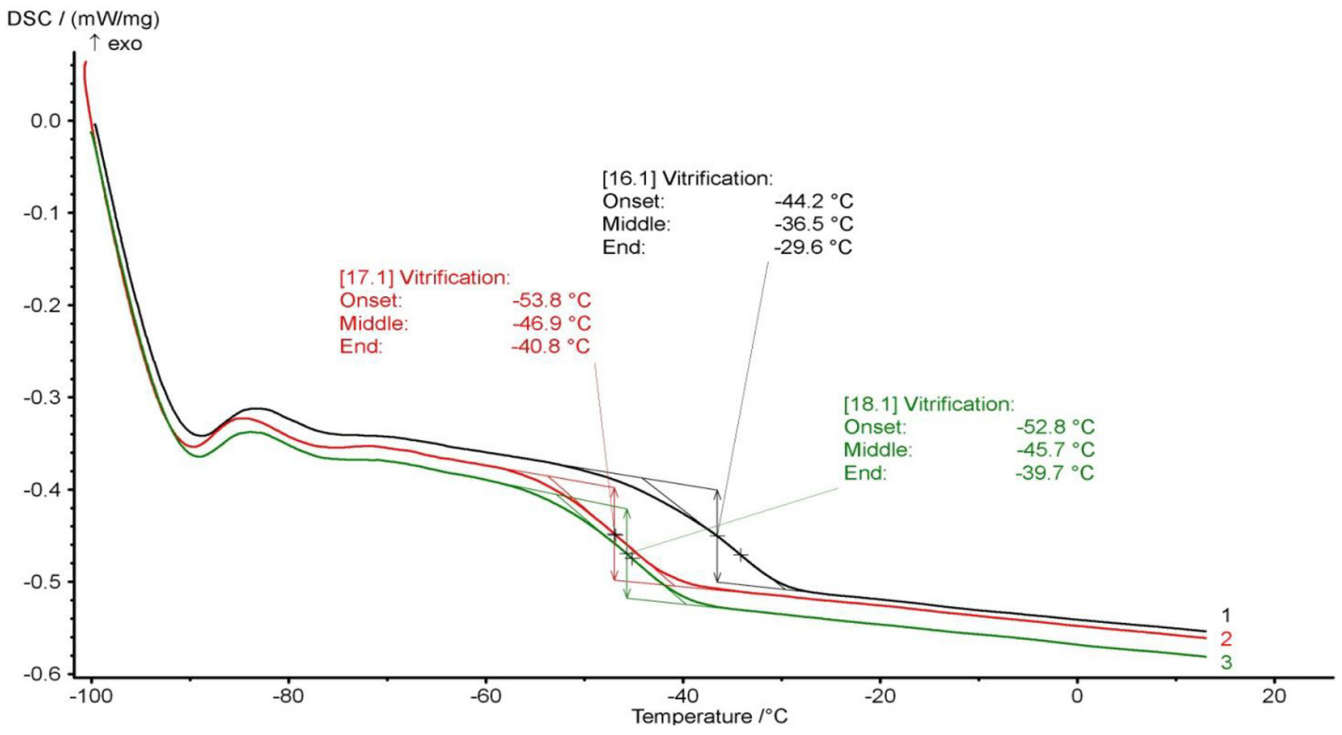

Рис. 2. Температуры стеклования образцов резин на основе БНКС-18 АМН: 1 - без пластификатора; 2 с содержанием 20 масс.ч. ДОТФ; 3 - с содержанием 20 масс.ч. ДОФ

Fig. 2. Glass transition temperatures of rubber samples based on BNKS-18 AMN: 1 - without plasticizer; 2 -with a content of $20 \mathrm{phr}$ DOTP; 3 - with a content of $20 \mathrm{phr}$ DOP

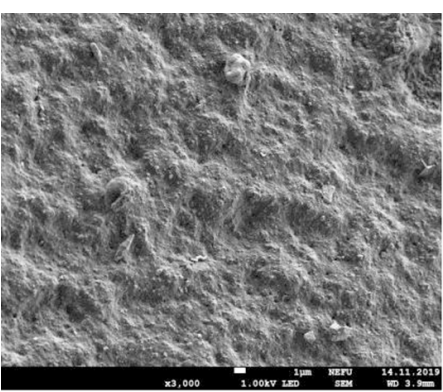

a)

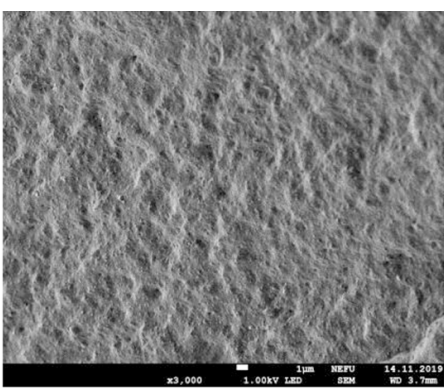

б)

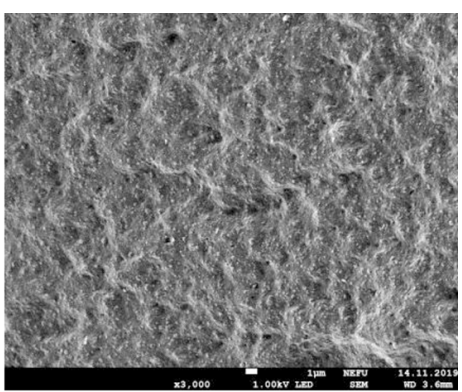

в)

Рис. 3. Микрофотографии поверхностей сколов (×3000) резин на основе БНКС-18 АМН: а - образец № 1 без пластификатора; б - образец № 4 - с содержанием 20 масс.ч. ДОФ; в - образец № 7 - с содержанием 20 масс.ч. ДОТФ

Fig. 3. Micrographs of the surfaces of chips $(\times 3000)$ of rubbers based on BNKS-18 AMN: a - sample no. 1 - without plasticizer; 6 - sample no. 4 - with a content of $20 \mathrm{phr}$ DOP; в - sample no. 7 - with a content of $20 \mathrm{phr}$ DOTP 
зе) исходного образца значительно отличается от поверхности пластифицированных образцов. Рельеф является недостаточно гладким, неоднородным, на нем наблюдаются отдельные частицы различной формы и размеров (при увеличении $\times 3000$ ), что свидетельствует о том, что распределение ингредиентов в резиновой смеси происходит неравномерно. По сравнению с исходным образцом поверхность пластифицированных образцов более однородна, не наблюдаются отдельные частицы и, соответственно, происходит более равномерное распределение ингредиентов.

Равномерность распределения ингредиентов в смесях является не только характеристикой технологических свойств получаемых композиций, но и тем показателем, от которого зависят эксплуатационные свойства изделий. Улучшение качества смешения ингредиентов резиновой смеси оказывает благоприятное влияние на все свойства. При динамических измерениях на приборе RPA-2000 можно определять энергию, запасаемую в полимере и обратимо отдаваемую им в каждом цикле. Мерой этой энергии служит модуль накопления (эластичности) G'. Одновременно определяется доля энергии, рассеянной в единице объема материала за цикл деформирования. Эта часть сопротивления материала деформированию характеризуется модулем потерь G". По результатам динамического испытания были построены кривые зависимости $\mathrm{G}^{\prime}$ и $\mathrm{G}^{\prime \prime}$ от частоты нагружения (рис. 4). На рис. 4А видно, что введение пластификаторов в резину приводит к снижению модуля накопления вследствие уменьшения межмолекулярного взаимодействия макромолекул каучука за счет экранирования пластификатором полярных групп полимерной матрицы [10-12]. Причем вклад в повышение подвижности надмолекулярных структур обоих пластификаторов (ДОФ и ДОТФ) практически одинаков. Показано, что у всех вулканизатов происходит рост $\mathrm{G}^{\prime}$ по мере увеличения частоты нагружения, т. е. происходит повышение сопротивления к циклическим воздействиям по мере роста частоты деформирования.
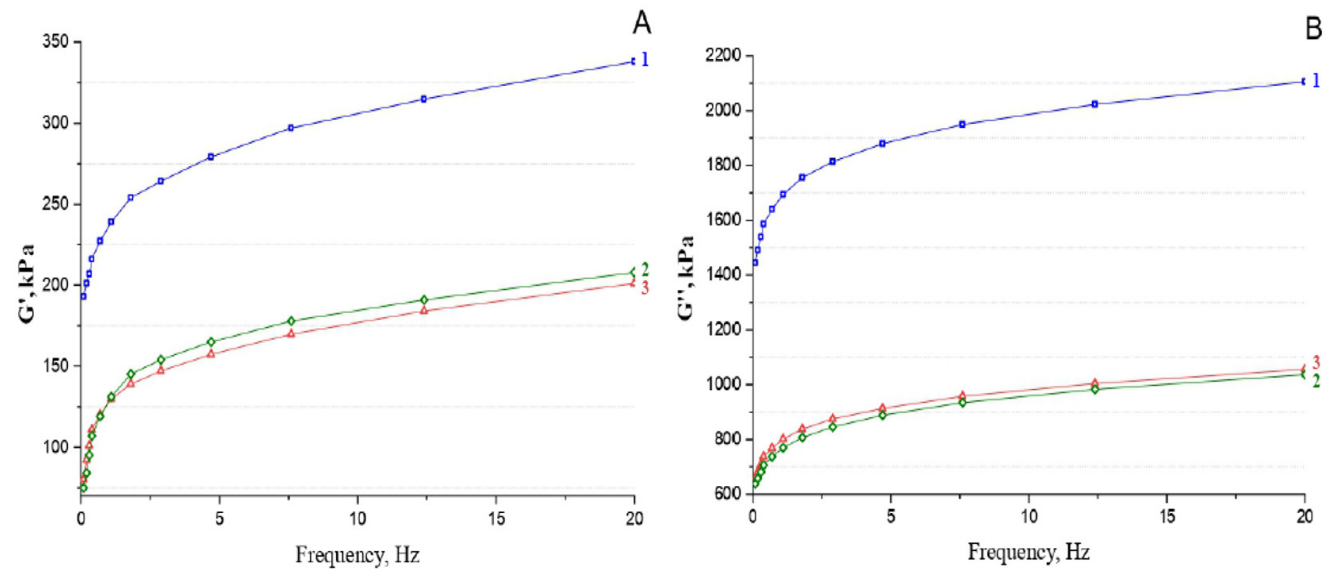

Рис. 4. Кривые зависимости модуля накопления (А) и модуля потерь (Б) резин на основе БНКС-18 АМН от частоты нагружения: 1 - без пластификатора; 2 - с содержанием 20 масс.ч. ДОФ; 3 - с содержанием 20 масс.ч. ДОТФ

Fig. 4. Curves of the accumulation modulus (A) and loss modulus (B) of rubbers based on BNKS-18 AMN on the loading frequency: 1 - without plasticizer; 2 - with a content of $20 \mathrm{phr}$ DOP; 3 - with a content of $20 \mathrm{phr}$ DOTP 
При рассмотрении зависимостей $\mathrm{G}^{\prime \prime}$ от частоты нагружения видно (рис. 4Б), что значения модуля потерь всех исследованных образцов резин с увеличением частоты деформирования повышаются за счет потери энергии при разрыве и преобразовании связей наполнительнаполнитель [13-15]. Причем отчетливо виден вклад пластификаторов в уменьшение сопротивления материала деформированию. Так образцы, содержащие ДОФ и ДОТФ, характеризуются низким модулем потерь, что можно объяснить уменьшением межмолекулярного взаимодействия между полярными группами бутадиен-нитрильного каучука при введении пластификаторов, вследствие чего повышается подвижность макромолекул.

\section{Выводы}

1. Введение пластификаторов ДОФ и ДОТФ в резиновые смеси на основе БНКС-18 АМН способствует получению резин с улучшенным комплексом эксплуатационных свойств, таких как эластичность, морозостойкость, стойкость к среде СЖР-3. При этом по результатам исследований выявлено, что введение пластификаторов в количестве 20 масс.ч. приводит к наиболее заметному улучшению таких показателей резин, как относительное удлинение при разрыве, стойкость к углеводородным средам и морозостойкость. По физико-механическим и низкотемпературным свойствам образцы, содержащие ДОТФ, заметно превосходят показатели образцов, содержащих ДОФ.

2. Сравнительный анализ полученных изображений методом РЭМ показывает, что по сравнению с исходным образцом, не содержащим пластификатор, поверхность пластифицированных образцов является более однородной, не наблюдаются отдельные частицы и, соответственно, происходит более равномерное распределение ингредиентов в каучуковой матрице.

3. Результаты исследования динамических свойств образцов резин показали, что введение пластификаторов в резину приводит к снижению модуля накопления $\left(\mathrm{G}^{\prime}\right)$ и модуля потерь $\left(\mathrm{G}^{\prime \prime}\right)$ вследствие уменьшения межмолекулярного взаимодействия макромолекул каучука за счет экранирования пластификатором полярных групп полимерной матрицы. Вклад обоих пластификаторов (ДОФ и ДОТФ) практически одинаков.

Таким образом, полученные нами данные показали перспективность применения ДОТФ в качестве альтернативы широко применяемому ДОФ.

\section{Благодарности / Acknowledgements}

Работа выполнена в рамках Госзадания Министерства науки и высшего образования РФ № AAAA-A17-117040710038-8.

This work was carried out within the framework of the State Assignment of the Ministry of Science and Higher Education of the Russian Federation No. AAAA-A17-117040710038-8.

\section{Список литературы / References}

[1] Резниченко С.В., Морозов Ю.Л. Большой справочник резинщика. Ч. 2. Резины и резинотехнические изделия. М.: ООО «Издательский центр «Техинформ» МАИ», 2012. 648 с. [Reznichenko S.V., Morozov Y.L. The great reference of rubberman. V. 2. Rubber and rubber products. Moscow, Techinform MAI, 2012. 648 p. (in Russian)] 
[2] Лазарева В.А., Кучугурный В.Е., Бортникова Е.А. Пластификаторы: маркетинговый обзор. Черкассы: ГП «Черкасский НИИТЭХИМ», 2011. 478 с. [Lazareva V. A., Kuchugurnyy V.E., Bortnikova E. A. Plasticizers: a marketing overview. Cherkassy, Cherkassy NIITEKHIM, 2011. 478 p. (in Russian)]

[3] Мамонов Р. А., Федотова Л. А., Печникова И. А., Потапченко Т. Д. Токсичность диоктилтерефталата. Токсикологический вестник, 2016, 6(153), 47-48. [Mamonov R. A., Fedotova L.A., Pechnikova I. A., Potapchenko T.D. Dioctylterephtalate toxicity. Toksikologcheskiy vestnik, 2016, 6(153), 47-48 (in Russian)]

[4] Соколова М. Д., Шадринов Н. В., Давыдова М. Л., Халдеева А.Р., Павлова В. В. Эластомерные материалы уплотнительного назначения для эксплуатации в условиях холодного климата России. Каучук и резина, 2018, 6, 402-409. [Sokolova M. D., Shadrinov N. V., Davydova M. L., Khaldeeva A.R., Pavlova V.V. Elastomeric materials for sealing purposes for operation in the cold climate of Russia. Kauchuk I Rezina, 2018, 6, 402-409 (in Russian)]

[5] Аммосова О.А., Аргунова А.Г., Ботвин Г.В. и др. Модифицированные полимерные и композиционные материалы для северных условий. Новосибирск: Изд-во СО РАН, 2017. 217 с. [Ammosova O. A., Argunova A. G., Botvin G. V. et al. Modified polymeric and composite materials for northern conditions. Novosibirsk, Izd-vo SO RAN, 2017, 217 p. (in Russian)]

[6] Тагер А. А. Физико-химия полимеров. М.: Научный мир, 2007. 576 c. [Tager A. А. Physicochemistry of polymers. M., Nauchnyy mir, 2007, 576 p. (in Russian)]

[7] Корнев А.Е., Буканова А. М., Шевердяев О.Н. Технология эластомерных материалов. М.: НППА «Истек», 2009. 504 с. [Kornev A.E., Bukanova А. M., Sheverdyaev O. N. Elastomeric material technology. M., Istek, 2009, 504 p. (in Russian)]

[8] Козлов П.В., Папков С.П. Физико-механические основы пластификации полимеров. М.: Химия, 1982. 224 с. [Kozlov P. V., Papkov S.P. Physical and mechanical bases of polymer plasticization. M., Khimiya, 1982, 224 p. (in Russian)]

[9] Барштейн Р.С., Кирилович В. И., Носовский Ю. Е. Пластификаторы для полимеров. М.: Химия, 1982. 200 с. [Barshtein R.S., Kirilovich V.I., Nosovskii Y.E. Plasticizers of polymers. M., Khimiya, 1982, 200 p. (in Russian)]

[10] Давыдова М. Л., Шадринов Н. В., Халдеева А.Р., Федорова А. Ф., Соколова М.Д. Влияние вулканизирующей системы на свойства и структуру резин на основе эпихлоргидринового каучука марки Hydrin T6000. Материаловедение, 2020, 9, 10-15. [Davydova M.L., Shadrinov N.V., Khaldeeva A.R., Fedorova A.F., Sokolova M.D. Influence of the vulcanizing system on the properties and structure of rubbers based on epichlorohydrin rubber brand Hydrin T6000. Materialovedenie, 2020, 9, 10-15 (in Russian)]

[11] Шайдаков В.В. Свойства и испытания резин. М.: Химия, 2002. 235 с. [Shaidakov V. V. Properties and testing of rubbers. M., Khimiya, 2002, 235 p. (in Russian)]

[12] Payne A. R., Whittaker R.E. Low strain dynamic properties of filled rubbers, Rubber Chem Technol, 1971, 44(2), 440-478.

[13] Fedorova A.F., Davydova M.L., $\quad$ Shadrinov N. V., $\quad$ Sokolova M.D., $\quad$ Pavlova V.V., Fedorov A.L. Investigation of the effect of plasticizer injection technology on the properties of epichlorohydrin rubbers, Journal of elastomers and plastics, First Published 30 Oct 2020.

[14] Robertson C.G., Roland C.M. Glass transition and interfacial segmental dynamics in polymer-particle composites, Rubber Chem Technol, 2008, 81(3), 506-522. 
[15] Bohm G. A., Tomaszewski W., Cole W., Hogan T. Furthering the understanding of the non linear response of filler reinforced elastomers, Polymer, 2010, 51(9), 2057-2068. 